\title{
Selective Inner-Filter on the Fluorescence Response of Chlorophyll and Pheophytin Molecules Extracted from Caesalpinia echinata Leaves
}

\author{
Jefferson M. S. Lopes, ${ }^{* a}$ Sanclayton G. C. Moreira ${ }^{a}$ and Newton M. Barbosa Neto ${ }^{\circledR a, b}$ \\ ${ }^{a}$ Programa de Pós-Graduação em Física, Instituto de Ciências Exatas e Naturais, \\ Universidade Federal do Pará, 66075-110 Belém-PA, Brazil \\ ${ }^{b}$ Programa de Pós-Graduação em Química, Instituto de Ciências Exatas e Naturais, \\ Universidade Federal do Pará, 66075-110 Belém-PA, Brazil
}

\begin{abstract}
Photophysical processes in multicomponent solutions composed by natural pigments, extracted from Caesalpinia echinata (Brazil-wood) leaves, are investigated. Beacuse of the main fluorescent molecules in these solutions are the chlorophyll a, chlorophyll $b$ and pheophytin a, all features are discussed regarding these three pigments. It is observed that the fluorescence bands of these macrocyclic molecules, located around ca. $673 \mathrm{~nm}$, are progressively quenched due to the increase of the solutions' concentration. Due to a significant overlapping between their fluorescence and absorption Q-bands, it is concluded that the inner-filter effect causes such quenching. Through analysis of steady-state and time-resolved fluorescence, it is verified that the inner filter acts more effectively on the emission assigned to chlorophyll a. Invoking reported extinction coefficients for these pigments in their isolated forms, we can give a qualitative interpretation of such selectivity considering the effectiveness of the overlapping between the absorption and fluorescence bands.
\end{abstract}

Keywords: inner-filter, fluorescence spectroscopy, multicomponent solutions, chlorophylls, deconvolution

\section{Introduction}

Flora is a vast source of natural pigments, most of them with great potential for applications in different areas such as photovoltaic and health sciences. ${ }^{1-3}$ Since these molecules are environment-friendly when compared to other artificial pigments, they stand out as possible substituents of well-established materials. Therefore, investigations on their structure, interaction mechanisms, ground, and excited state properties, and others are crucial to inspire and improve the potential uses of such molecules.

Various methods of extraction of pigments from plants have been developed, ${ }^{4,5}$ however, until nowadays, the most straightforward and cheapest procedure is based on the emulsion of leaves in organic solvents. ${ }^{6}$ This method, nonetheless, lacks selectivity and is very dependent on leaf-solvent interactions ${ }^{7}$ and the content of pigments in each leaf species. ${ }^{8,9}$ Such aspects imply that a great variety of pigments is released together in the solvent, resulting in multicomponent solutions. ${ }^{6}$ According to the literature, the main classes of pigments available in leaves are the

*e-mail: lopesjefferson01@yahoo.com.br carotenoids and chlorophylls, both of them receiving considerable attention from the scientific community due to their photophysical, ${ }^{10,11}$ photochemical,,${ }^{11,12}$ and optical properties. ${ }^{13-15}$

Briefly, the carotenoids are pigments composed by a linear conjugated chain of around forty carbon atoms that due to their high conjugation length, present absorption and fluorescence bands spread over the near-UV and visible spectral region. ${ }^{11,16}$ The chlorophylls are tetrapyrrole macrocyclic rings stabilized at its central position by a magnesium ion $\left(\mathrm{Mg}^{2+}\right)$, being the ring decorated with outlying substituents (e.g., phytol). These pigments, which are classified as natural metalloporphyrins, exist in six different structures known as chlorophylls a, b, $c_{1}, c_{2}, d$, and $\mathrm{f}$. The two first types are the most commonly found in plants. ${ }^{17}$ The substitution of the central metallic ion of chlorophyll by two hydrogen atoms results in the free base type structure, named pheophytin, which is also commonly found in vegetables. ${ }^{10,17}$ Qualitatively, the photophysical properties of chlorophylls and pheophytins are explained by the four-orbital model proposed by Gouterman. ${ }^{10,18}$ According to this model, their absorption spectrum is described by transitions from the ground state to the first 
(Q-band) and second (Soret-band) excited states. Their luminescence is understood regarding the relaxation process from their first excited state, which results in significant fluorescence bands in the red portion of the spectrum. ${ }^{10}$

At first glance, the non-selectivity of the emulsion method for extraction of pigments might seem an undesirable issue. However, the obtained molecules can synergistically interact one each other, giving rise to solutions with unique features, regarding their photophysical and photochemical properties. Here, we investigate the fluorescence emitted by multicomponent solutions composed of pigments extracted from fresh Caesalpinia echinata (Brazil-wood) leaves emulsified in methanol. We observed that the inner-filter affects significantly the fluorescence bands located around ca. $673 \mathrm{~nm}$ as the concentration of extracted chlorophyll a (Chl a), chlorophyll b (Chl b) and pheophytin a (Pheo a) molecules increase. Using Voigt deconvolution of the steady-state spectrum and time-resolved fluorescence signatures, we verified that this effect is selective, acting mostly on the emission assigned to $\mathrm{Chl}$ a. This behavior is not straightforward since the fluorescence features of $\mathrm{Chl} b$ and Pheo a are expected to be affected too. We propose a qualitative model to explain this selectivity on the observed inner-filter.

\section{Experimental}

The pigments were extracted from fresh leaves of Brazil-wood (Caesalpinia echinata) at room temperature (ca. $23^{\circ} \mathrm{C}$ ), by the emulsion of $1.0 \mathrm{~g}$ of cut leaves in $35 \mathrm{~mL}$ of methanol (MeOH), used as received from Sigma-Aldrich (Saint Louis, USA). Before extraction of the pigments, the leaves were washed with distilled water and subsequently dried. The leaves were cut in strips of similar shapes and area of around $0.5 \mathrm{~cm}^{2}$. During the extraction, the solution was stirred by a magnetic bar to ensure the homogeneity of the solution.

Stepwise extraction was performed during $240 \mathrm{~min}$, where 12 aliquots of $2.0 \mathrm{~mL}$ were removed at every $20 \mathrm{~min}$ from the first bath and stored in a vial filled with extra $2 \mathrm{~mL}$ of solvent. This additional dissolution was adopted to avoid saturation problems during the acquisition of the absorption spectra. Before storing the aliquots into the vial, the samples were filtered by a filter paper method aiming to discard undesired solid residues.

UV-Vis absorption spectra were acquired in a Jasco spectrophotometer, model V-670. Steady-state fluorescence measurements were performed exciting the samples with a continuous wave $(\mathrm{CW})$ laser (excitation wavelength at $473 \mathrm{~nm}$ ) and detecting the photoluminescence signal in a $90^{\circ}$ configuration with a portable spectrophotometer from Ocean Optics, model USB 2000. Time-resolved fluorescence signal was acquired using a time-correlated single photon counting system from Horiba, model Delta Flex, with 27 ps of temporal resolution. The excitation source employed was a pulsed light emitting diode (LED) with $8 \mathrm{MHz}$ of repetition rate and excitation wavelength at $352 \mathrm{~nm}$. All decays were probed at $673 \mathrm{~nm}$. The spectroscopic measurements were performed at room temperature (ca. $23^{\circ} \mathrm{C}$ ), and the solutions were placed in a quartz cuvette with a $1 \mathrm{~cm}$ pathlength and four polished windows.

\section{Results and Discussion}

Absorption spectroscopy: second-derivative analysis, spectral deconvolution, and assignments of pigments

In Figure 1, we present the absorption spectra of the twelve aliquots obtained along $2 \mathrm{~h}$ of an emulsion prepared with leaves in methanol. From the spectra, it is verified that a great variety of different pigments is released in the solutions. A set of bands are observed in the spectral region below $400 \mathrm{~nm}$ and are attributed to electronic transitions of terpenoids ${ }^{19}$ and flavonoids. ${ }^{20,21}$ In fact, according to Bastos et al. ${ }^{22}$ Caesalpinia echinata leaves contain a high amount of these two classes of phenolic compounds. Unfortunately, we were not able to distinguish these molecules with the optical signatures presented here, and an accurate assignment is still elusive. Therefore, we focus our analysis on the bands located in the region of $400-750 \mathrm{~nm}$, related to chlorophylls and carotenoids. ${ }^{17}$

Due to the multicomponent character of the solutions, formed along the extraction, intermolecular interactions can be favored implying in the rise of novel bands in the spectrum. Then, the assignment of the absorption bands of the mixture must be performed carefully. The normalization of the absorption spectra by their respective absorbance values at $412 \mathrm{~nm}$ reveals that the bands in the UV-Vis spectral region are not modified along the extraction, indicating that new species are not formed, see Figure S1 in the Supplementary Information (SI) section.

Considering previously reported assignments,,$^{10,17}$ in Figure 1 we can identify the Soret bands of Pheo a, $\mathrm{Chl} \mathrm{a}$ and $\mathrm{Chl} \mathrm{b}$ at 412, 437, and $469 \mathrm{~nm}$, respectively. A minor contribution coming from pheophytin $b$ (Pheo b) is expected to occur between the Soret bands of $\mathrm{Chl} b$ and Pheo $b,{ }^{17}$ however, no signal of this band is detected. Since features assigned to other structures, such as pheophorbide and chlorophyllins are not observed in our solutions, we assumed that the only chlorophyll-like molecules obtained along the extraction, with significant concentration, are 
these three mentioned pigments. From the assignments of the Soret bands (412 nm for Pheo a, $437 \mathrm{~nm}$ for Chl a and $469 \mathrm{~nm}$ for $\mathrm{Chl} \mathrm{b}$ ), it is observed that the rates of extraction for the pigments are unchanged, presenting Pheo a the more significant rate $\left(0.01 \mathrm{~min}^{-1}\right)$, followed by $\mathrm{Chl} \mathrm{a}\left(0.008 \mathrm{~min}^{-1}\right)$ and Chl b $\left(0.006 \mathrm{~min}^{-1}\right)$, see Figure S2 (SI).

Four bands, centered respectively at 507, 536, 610, and $663 \mathrm{~nm}$ are observed in the chlorophylls' Q-band spectral region. The attributions of these bands are not straightforward because some transitions in the Q-band of both chlorophylls considered and Pheo a can be overlapped between each other in the interval of 600-750 nm. ${ }^{17}$

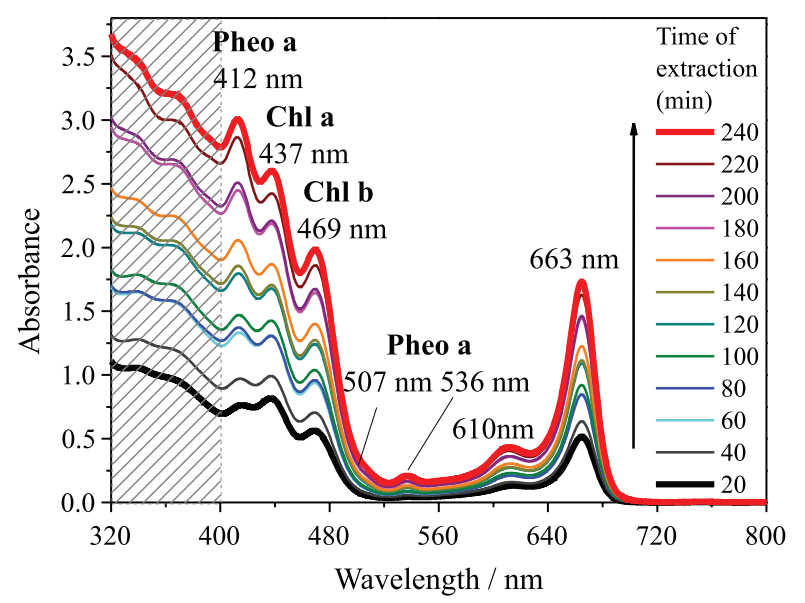

Figure 1. Absorbance spectra of the aliquots taken at different times from the emulsion of Caesalpinia echinata (Brazil-wood) leaves in methanol. Attributions of the Soret bands of Pheo a (412 nm), Chl a (437 nm) and Chl b (469 nm), as well as some of the unambiguous Q-bands of Pheo a (at 507 and $536 \mathrm{~nm}$ ), are emphasized. The dashed area below $400 \mathrm{~nm}$ indicates the region related to the phenolic compounds.

According to the Gouterman's model, ${ }^{10}$ the absorption bands of cyclic tetrapyrroles are due to transition dipoles oriented in two perpendicular directions of the macrocyclic ring. When a metal ion is placed in the ring's central positions, the transitions in both directions become degenerate. ${ }^{10,18}$ This is the case for chlorophylls, where only one electronic transition and its vibronic counterpart form their Q-band. ${ }^{10}$ However, when two hydrogen atoms substitute the $\mathrm{Mg}^{2+}$ ion, during the formation of pheophytins, the degeneracy of the electronic transitions is broken and the Q-band displays transitions occurring in the two distinct directions of the macrocyclic ring. ${ }^{10}$ Following these considerations and the assignments previously proposed, ${ }^{17,23}$ the main Q-bands of $\mathrm{Chl}$ a and $\mathrm{Chl}$ b dissolved in methanol, are expect to be located in ca. $663 \mathrm{~nm}$ and ca. $649 \mathrm{~nm}$, respectively. Both electronic bands might present their respective vibronic bands at around $610 \mathrm{~nm}$ and $605 \mathrm{~nm} .{ }^{17}$ In the case of Pheo a (dissolved in methanol), four bands have been previously reported ${ }^{17}$ at around 507,
536,610 and $665 \mathrm{~nm}$. These attributions confirm that from 600 to $750 \mathrm{~nm}$ the bands related to all these three pigments might be superposed between each other in our spectra.

Aiming to obtain a more accurate assignment of the absorption bands, we performed second-derivative analysis and deconvolution with Voigt functions on the absorption spectrum of the last aliquot, i.e., obtained after $240 \mathrm{~min}$ of an emulsion of leaves in methanol. It is worth to mention that Voigt functions are employed because their more general characteristics when compared with Gaussian and Lorentzian functions. ${ }^{24}$ As shown in Figure 2, the Voigt functions centered at 437, 610 and $663 \mathrm{~nm}$, present correspondence with the inflection points of the secondderivative spectrum, depicted in the bottom panel of Figure 2 and are assigned to $\mathrm{Chl} \mathrm{a.} \mathrm{For} \mathrm{Chl} b$, the assigned curves are centered at 469 and $649 \mathrm{~nm}$. Our method does not allow us to resolve the vibronic Q-band of this molecule, at around $605 \mathrm{~nm}$. Because of this, we are assuming that this feature is superposed with the Chl a's Voigt curve at $610 \mathrm{~nm}$. It is important to highlight that the existence of the transition at $649 \mathrm{~nm}$ is not directly indicated by the second-derivative analysis since there is no inflection point at this wavelength. However, we infer its existence considering the asymmetric shape of the peak at $663 \mathrm{~nm}$ (for better visualization, see Figure S3, SI). Considering that Voigt distributions are symmetric around their central positions, the occurrence of asymmetry in its secondderivative peaks indicates the existence of hidden peaks, in other words, two bands with very close centers but with different magnitudes. Contributions of Pheo a are related to the Voigt curves centered at 412, 507, 536 and $665 \mathrm{~nm}$. The vibronic progression of the electronic transition at $665 \mathrm{~nm}$ in the Q-band is expected to be located at around $610 \mathrm{~nm}$ and so is assumed to be overlapped with the Chl a's transition at $610 \mathrm{~nm}$. Also, since the second-derivative peak at ca. $469 \mathrm{~nm}$ is very asymmetric (for a better visualization see Figure S3, SI), we propose the existence of an extra Voigt curve centered slightly higher than this value. As shown in Figure 2, by deconvolution analysis we obtain a Voigt curve centered at $484 \mathrm{~nm}$. Following the literature, this feature can be assigned to the absorption band of some carotenoid related molecule. ${ }^{16}$ Unfortunately, by absorption spectroscopy we are not able to elucidate exactly which specific carotenoid this curve is related.

Steady-state and time-resolved fluorescence of chlorophylls and pheophytin a molecules: description of the selective inner-filter

Although we have extracted a great variety of molecules from Caesalpinia echinata leaves, the main contribution in 


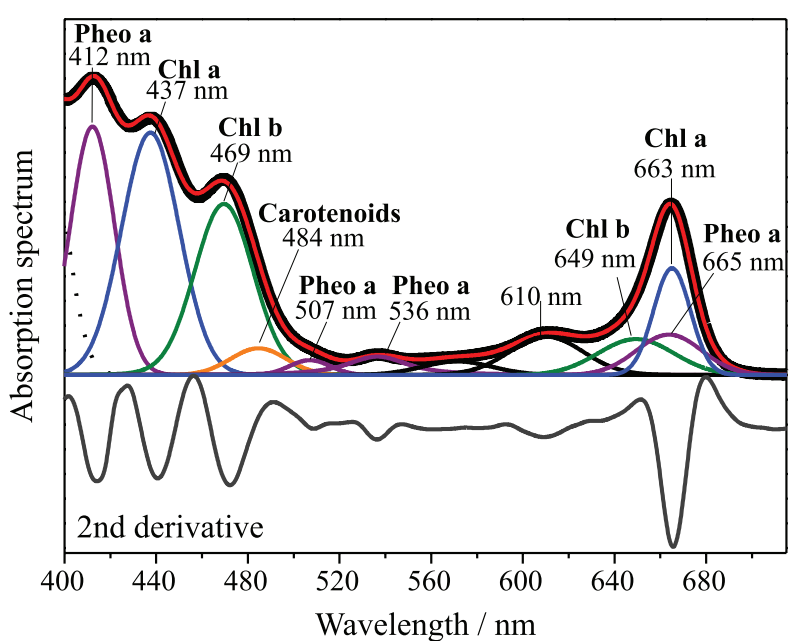

Figure 2. Voigt deconvolution (solid red line) and second derivative analysis (solid grey line at the bottom panel) applied to the absorption spectrum (solid black line) of the aliquot obtained after $240 \mathrm{~min}$ of an emulsion of leaves in methanol. The Voigt curves are attributed to the transitions of Pheo a (solid purple lines), Chl a (solid blue lines) and Chl b (solid green lines). The curve at $610 \mathrm{~nm}$ is interpreted as a superposition of the vibronic Q-bands of these three molecules. The curve at $484 \mathrm{~nm}$ (solid orange line) is assigned to carotenoids released in the solution.

the fluorescence spectrum of the aliquots comes from the chlorophylls (a and b) and Pheo a, as shown in Figure 3. This occurs because the fluorescence quantum yield of these macrocyclic structures, although small, ${ }^{10,17,25} \mathrm{ca} \cdot 10^{-2}$, is still much higher when compared to other obtained luminescent molecules such as carotenoids, ${ }^{25,26}$ which present values of the order of $10^{-4}$. As shown in Figure S4 (SI), we verified just minor contributions due to carotenoids, located at around 515 and $562 \mathrm{~nm}$, in the fluorescence spectrum of the most concentrated aliquot. No accurate assignment is obtained for the band at ca. $515 \mathrm{~nm}$ but, following previous reports, the band at ca. $562 \mathrm{~nm}$ can be due to $\beta$-carotene molecules. ${ }^{25,26}$ Because the carotenoid's signal is very low, we base all discussions in this work on the fluorescence features of Chl a, Chl b and Pheo a.

In Figure 3a, we compare the normalized absorption and fluorescence spectra (excitation at $473 \mathrm{~nm}$ ) of the aliquot obtained after $20 \mathrm{~min}$ of an emulsion of leaves in methanol. A significant overlapping between the Q-absorption and the fluorescence bands of chlorophylls and Pheo a is observed at around $673 \mathrm{~nm}$. In Figure 3b, we show the evolution of the normalized fluorescence spectrum along the extraction period. It is observed that the fluorescence bands, located in the region where occurs the overlap with absorbance spectrum (ca. $673 \mathrm{~nm}$ ), are considerably quenched as the amount of extracted molecules increases. Moreover, an apparent relative increase of the bands located around ca. $720 \mathrm{~nm}$ as well as a redshift from 673 to $683 \mathrm{~nm}$ for the maxima of the fluorescence spectrum is also noted. Since the fluorescence bands become increasingly affected by the extraction time, i.e., by the increase in the concentration of pigments in the aliquots, we attribute all these behaviors to the inner-filter effect, which are triggered by the increase in the absorbance values at the overlapped region. As we will discuss later, a complex scenario takes place in our multicomponent aliquots, since the three emitters identified here act as quenchers for one each other as well as to themselves through the reabsorption mechanism. ${ }^{27-30}$

In order to explore in more details the roles of both chlorophylls (a and b) and Pheo a in the inner-filter effect, we performed Voigt deconvolution on the fluorescence spectra of the least $(20 \mathrm{~min})$ and the most $(240 \mathrm{~min})$ concentrated aliquots. As depicted in Figures $4 \mathrm{a}$ and $4 \mathrm{~b}$,
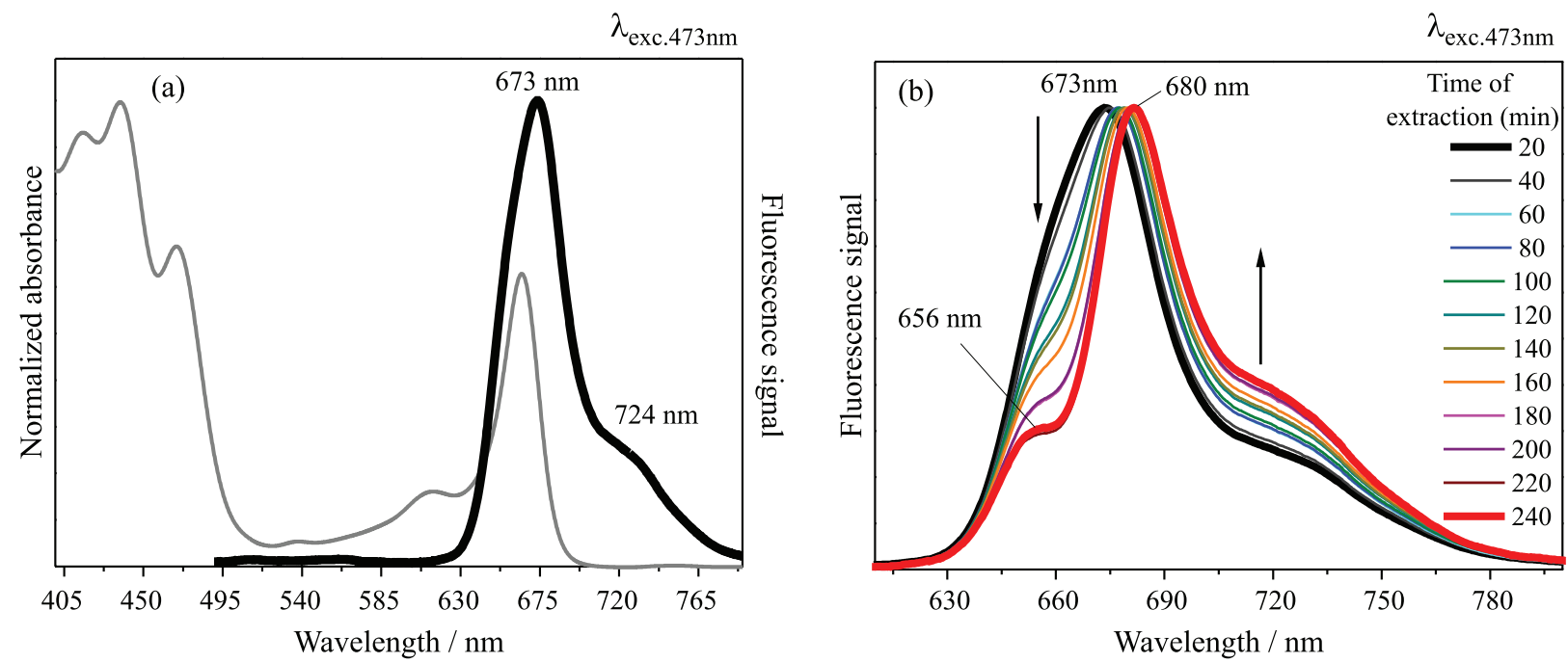

Figure 3. (a) Comparison between absorption (solid gray line) and fluorescence spectra (solid black line) (excitation at $473 \mathrm{~nm}$ ) of the aliquot obtained after $20 \mathrm{~min}$ of an emulsion of leaves in methanol. (b) Evolution of the fluorescence spectrum (excitation at $473 \mathrm{~nm}$ ) of aliquots acquired at intervals of $20 \mathrm{~min}$. The changes in the relative intensity of bands around $653 \mathrm{~nm}$ indicate the occurrence of the inner-filter effect. 
both spectra are well fitted adopting six Voigt functions, located at 653, 673, 683, 700, 720, and $745 \mathrm{~nm}$. Assuming previous assignments, the Voigt curves at 653,673 and $683 \mathrm{~nm}$ are attributed to the $\mathrm{Q}(0,0)$ fluorescence bands of $\mathrm{Chl} \mathrm{b,} \mathrm{Chl} \mathrm{a,} \mathrm{and} \mathrm{Pheo} \mathrm{a,} \mathrm{respectively.}{ }^{17,31-35}$ The other three bands $(700,720$, and $745 \mathrm{~nm})$ are respectively assigned to the vibronic fluorescence bands $\mathrm{Q}(0,1)$ of these pigments following the same order.

Besides the central position of the bands, we support our analysis on the percentage contribution of each Voigt function to the total area of the normalized fluorescence spectra. This parameter is adopted because the area of an emission band is related to its corresponding quantum yield, which is very sensitive to the inner-filter. ${ }^{36,37}$ As presented in Table 1, a significant decrease in the percentage contribution of the $\mathrm{Q}(0,0)$ fluorescence band of Chl a (at $673 \mathrm{~nm}$ ) is verified, since this value changes from 43 to $8 \%$. The contribution assigned to $\mathrm{Chl} \mathrm{b}$ (at $653 \mathrm{~nm}$ ) present minor change, going from 13 to $11 \%$. Contrarily, the contribution of Pheo a to the fluorescence spectrum increased from 17 to $34 \%$. In the case of the vibronic bands, it is verified an increase in the sum of their percentage contributions when we compare the first and the last aliquots, which furnish additional evidence of the inner-filter effect.

Following these results, the apparent shift presented in Figure 3b, can be interpreted assuming that in the least concentrated aliquot, the emission band of $\mathrm{Chl}$ a is the most intense, but after the occurrence of the inner-filter effect, the Pheo a's assumes this condition. This means that, no shift would be taking place at all, but a redistribution of the most intense emission bands, related to $\mathrm{Chl}$ a and Pheo a. Because these changes are so much more significant in the fluorescence of $\mathrm{Chl}$ a in comparison to the other pigments, this analysis suggests the existence of a selective inner-filter effect. This behavior is not expected, mainly because the bands of $\mathrm{Chl} b$ and Pheo a are also located in the overlapped area.

Beyond changes in the steady-state fluorescence spectrum, the inner-filter effect is expected to diminish the total rate of deactivation from the excited state of a molecule. ${ }^{36,37}$ In other words, this effect increases the excited state lifetime. ${ }^{36,37}$ Probing the fluorescence lifetime of all aliquots, at $673 \mathrm{~nm}$, we observe that the decay follow

Table 1. Parameters obtained after deconvolution with Voigt functions of the fluorescence spectra of the least $(20 \mathrm{~min})$ and the most $(240 \mathrm{~min})$ concentrated aliquots

\begin{tabular}{lccc}
\hline $\begin{array}{l}\text { time of } \\
\text { extraction } / \text { min }\end{array}$ & $\lambda^{\mathrm{a} / \mathrm{nm}}$ & Assignment & Area $^{\mathrm{b}} \%$ \\
\hline & 653 & $\mathrm{Q}(0,0) \rightarrow$ Chl b & 13 \\
& 673 & $\mathrm{Q}(0,0) \rightarrow$ Chl a & 43 \\
& 683 & $\mathrm{Q}(0,0) \rightarrow$ Pheo b & 17 \\
& 700 & $\mathrm{Q}(0,1) \rightarrow$ Chl b & 4 \\
& 720 & $\mathrm{Q}(0,1) \rightarrow$ Chl a & 16 \\
& 745 & $\mathrm{Q}(0,1) \rightarrow$ Pheo a & 7 \\
\hline 60 & $\mathrm{Q}(0,0) \rightarrow$ Chl b & 11 \\
& 653 & $\mathrm{Q}(0,0) \rightarrow$ Chl a & 8 \\
& 673 & $\mathrm{Q}(0,0) \rightarrow$ Pheo b & 34 \\
& 683 & $\mathrm{Q}(0,1) \rightarrow$ Chl b & 13 \\
& 700 & $\mathrm{Q}(0,1) \rightarrow$ Chl a & 20 \\
& 720 & $\mathrm{Q}(0,1) \rightarrow$ Pheo a & 13
\end{tabular}

${ }^{\mathrm{a} C e n t r a l}$ position of each Voigt curve; ${ }^{\mathrm{b}}$ percentage contribution to the total area of the spectrum.
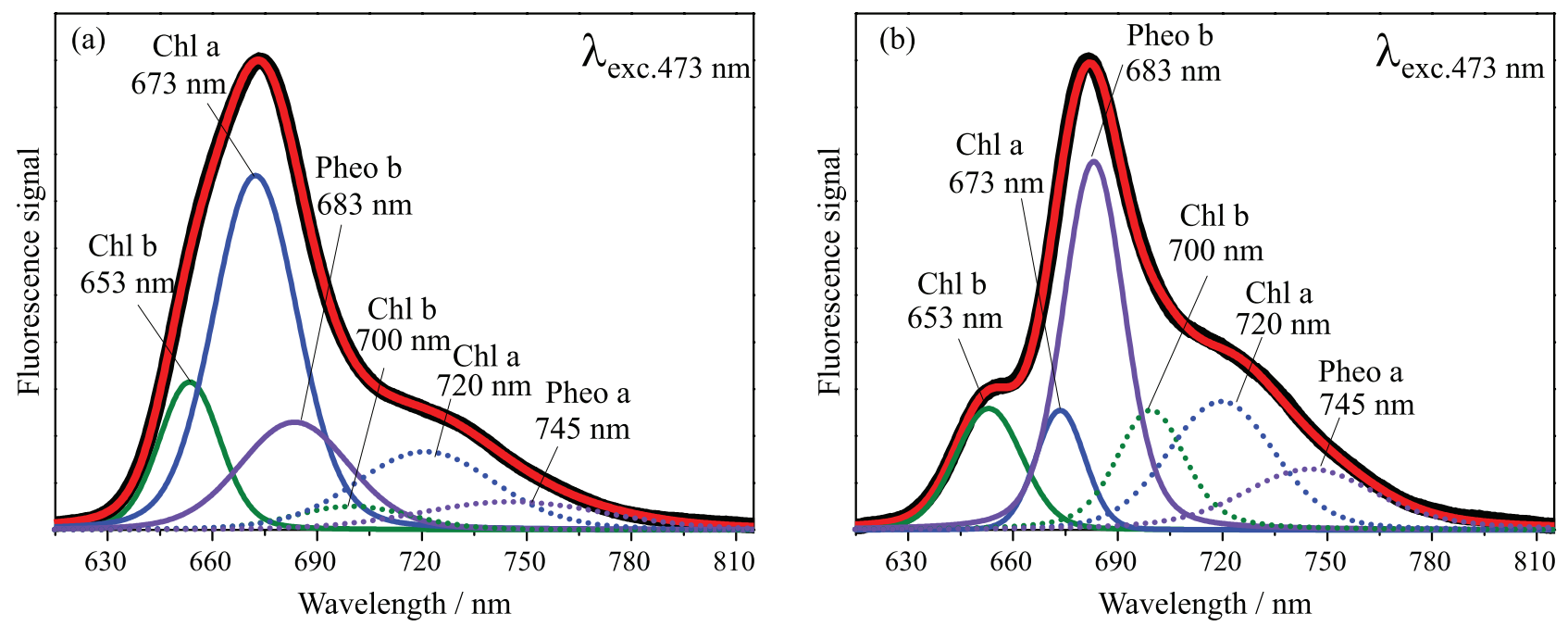

Figure 4. Fluorescence spectrum (solid black line) and theoretical Voigt-based deconvolution (solid red line) of (a) the least (20 min) and (b) the most $(240 \mathrm{~min}$ ) concentrated aliquots. The samples were excited at $473 \mathrm{~nm}$. The bands at $653 \mathrm{~nm}$ (solid green line), $673 \mathrm{~nm}$ (solid blue line) and $683 \mathrm{~nm}$ (solid purple line) are attributed to $\mathrm{Q}(0,0)$ fluorescence bands from $\mathrm{Chl} \mathrm{b}, \mathrm{Chl}$ a, and Pheo a, respectively. The bands at $700 \mathrm{~nm}$ (dotted green line), $720 \mathrm{~nm}$ (dotted blue line) and $745 \mathrm{~nm}$ (dotted purple line) are attributed to the respective $\mathrm{Q}(0,1)$ fluorescence bands from these pigments. 
bi-exponential kinetics, see Figure 5a. For the first aliquot, characteristic lifetimes of $\tau_{1}=2.35 \mathrm{~ns}$ and $\tau_{2}=5.79 \mathrm{~ns}$, with respective percentage contributions of ca. $6 \%$ and ca. $94 \%$, were obtained. The lifetimes of 2.35 and 5.79 ns can be assigned to chlorophylls $b$ and a, respectively. ${ }^{34,38,39}$ As pointed in the literature, in many solvents and concentration conditions, the excited state lifetime of Pheo a is usually very close to the value found for $\mathrm{Chl}$ a, being ca. $5.79 \mathrm{~ns}$ in $n$-hexane. ${ }^{17,40}$ Assuming these reported results, we have tried to fit the decay kinetic with three exponential functions, but unfortunately, we could not distinguish between the decay of $\mathrm{Chl}$ a and Pheo a. Because of that, all considerations concerning the $\tau_{2}$ lifetime can be assigned to contributions from $\mathrm{Chl}$ a as well as from Pheo a molecules.

Evaluation of the decay kinetics of posterior aliquots reveals a progressive increase on the lifetime $\tau_{2}$, up to $6.14 \mathrm{~ns}$, corresponding to a variation of ca. $350 \mathrm{ps}$, as the aliquots become more concentrated, i.e., as the inner-filter effect becomes more effective, see Figure $5 \mathrm{~b}$ and Table 2. No changes in the $\tau_{1}$ lifetime as well as in the percentage contributions of each decay were verified. This result together with the analysis of the percentage area of Voigt curves obtained for the emission spectra shows that the inner-filter is selective and affects mostly the excited states of Chl a molecules.

The mechanisms behind the inner-filter effect can be understood in two ways, described as the following: (i) in the overlapped region, each molecule can affect themselves by the reabsorption, ${ }^{27-30}$ or (ii) the fluorescence bands of each molecule can be influenced by the absorption bands of other molecules present in the solution. ${ }^{36,37}$ In both cases, the effectiveness of this photophysical process is dependent on the absorbance values at the overlapped region, ${ }^{36,37}$ which are defined
Table 2. Fluorescence lifetime parameters obtained by bi-exponential deconvolution of the decay signal

\begin{tabular}{lccc}
\hline $\begin{array}{l}\text { Time of } \\
\text { extraction } / \min \end{array}$ & $\tau_{1}(\mathrm{Chl} \mathrm{b})^{\mathrm{a}} / \mathrm{ns}$ & $\begin{array}{c}\tau_{2}(\mathrm{Chl} \mathrm{a} / \mathrm{Pheo} \mathrm{a})^{\mathrm{b}} / \\
\mathrm{ns}\end{array}$ & $\Delta \tau_{2}^{\mathrm{c}} / \mathrm{ps}$ \\
\hline 20 & 2.35 & 5.79 & 0 \\
40 & 2.35 & 5.79 & 0 \\
60 & 2.35 & 5.79 & 0 \\
80 & 2.35 & 5.79 & 0 \\
100 & 2.35 & 5.83 & 40 \\
120 & 2.35 & 5.87 & 80 \\
140 & 2.35 & 5.95 & 170 \\
160 & 2.35 & 6.02 & 230 \\
180 & 2.35 & 6.05 & 260 \\
200 & 2.35 & 6.07 & 280 \\
220 & 2.35 & 6.11 & 320 \\
240 & 2.35 & 6.14 & 350 \\
\hline
\end{tabular}

${ }^{a}$ Lifetime attributed to chlorophyll $b$; ' bifetime attributed to chlorophyll a and pheophytin a; "change of the $\tau_{2}$ along the time of extraction of pigments.

as the product between the corresponding extinction coefficient and the concentration of each pigment. In Figure 6, we compare the deconvoluted absorption and fluorescence spectra of the least concentrated aliquot. We observe that the absorption bands of $\mathrm{Chl} \mathrm{a,} \mathrm{Chl} \mathrm{b}$ and Pheo a can both affect one each other as well as themselves. The fluorescence band of $\mathrm{Chl} \mathrm{b}$ (centered at $653 \mathrm{~nm}$ ) is overlapped with the blue-wing part of the absorption bands of Pheo a and $\mathrm{Chl}$ a molecules as well as with its own absorption band. A similar trend is observed for the other molecules, see Figure 6. In this scenario, the inner-filter effect should be efficient in the fluorescence response of all these molecules.
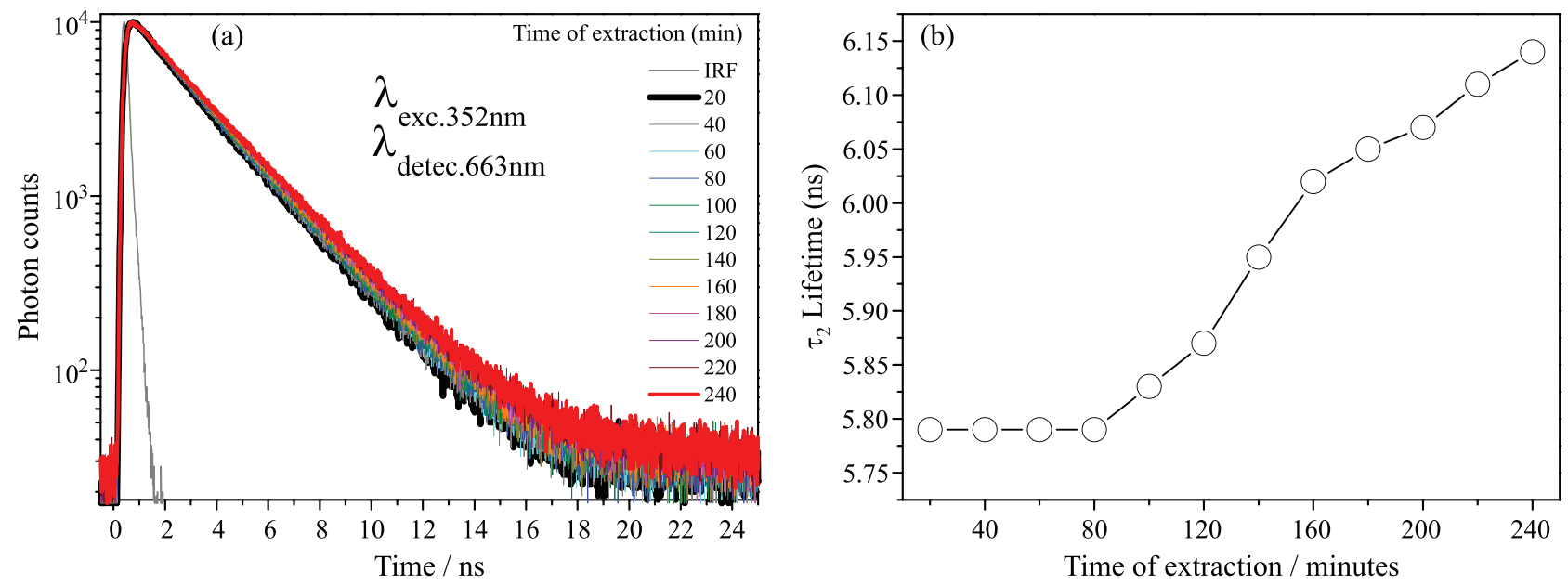

Figure 5. (a) Fluorescence lifetime of the aliquots acquired at different times of emulsion of leaves in methanol. Samples were excited at $352 \mathrm{~nm}$, and the decays are detected at $673 \mathrm{~nm}$. (b) Increase in the $\tau_{2}$ lifetime along the time of acquisition of the aliquots. 
Supporting our interpretation on the concentration and extinction coefficients at the overlapped bands, we propose a possible explanation for the observed selectivity of the inner-filter effect. Assuming that at a fixed wavelength, the variation in the absorbance spectrum is directly proportional to the change in the concentration, we verified increases of order of ca. 4.0, ca. 3.1 and ca. 3.5 times in the absorbance of the Soret bands of Pheo a $(412 \mathrm{~nm})$, Chl a (437 nm) and Chl b (469 nm), respectively. The absorbance of the Soret bands is considered in this discussion because only in this region we can distinguish unequivocally the contributions coming from these three molecules. Qualitatively, we observe that the concentration variation is almost the same for all these three pigments, reinforcing the expectation that the inner-filter should occur almost equally in all these three molecules. On the other hand, as described in the literature, the extinction coefficient of $\mathrm{Chl} \mathrm{b}$ is ca. 3.5 times smaller than the values for $\mathrm{Chl} \mathrm{a}$ and Pheo a molecules, at around $655 \mathrm{~nm}$, in methanol environment. ${ }^{17,41}$ Since the absorption of $\mathrm{Chl}$ a and Pheo a acts more effectively on the emission of $\mathrm{Chl}$ a and $\mathrm{Chl} \mathrm{b}$, while the fluorescence of $\mathrm{Chl} b$ is more effectively quenched by reabsorption mechanism, ${ }^{27-30}$ we believe that the combination of factors like molecular concentration, extinction-coefficients, and band overlap can explain the selectivity of the inner-filter effect. Note that, due to the relative lower extinction coefficient presented by the $\mathrm{Chl} \mathrm{b}$, in this region, its absorbance would not achieve sufficient values to affect the fluorescence response of the other molecules in the aliquots.

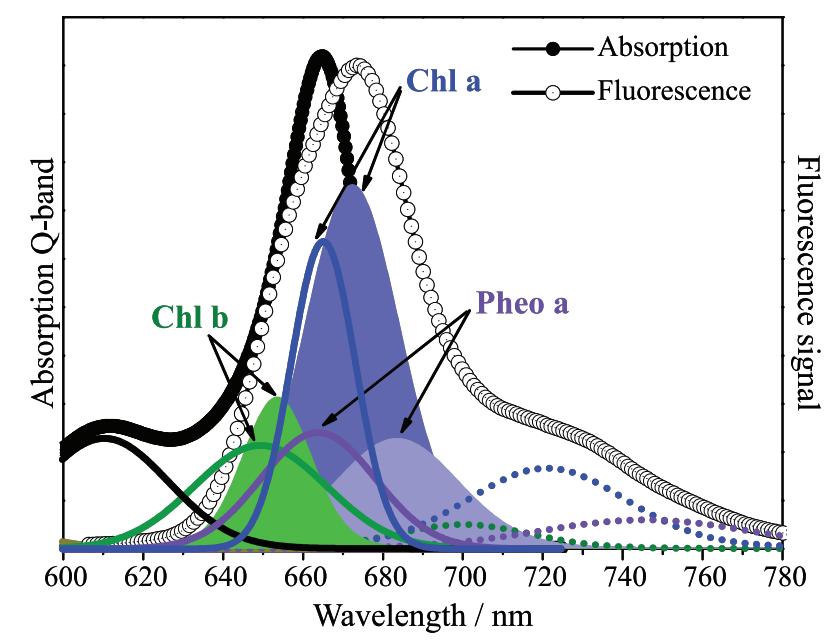

Figure 6. Overlapping between the deconvoluted absorption (full circles) and fluorescence (open circles) spectra of the least concentrated aliquot, acquired after $20 \mathrm{~min}$ of an emulsion of leaves in methanol. The Voigt curves with filled area represent the fluorescence bands of $\mathrm{Chl} b$ (green), $\mathrm{Chl}$ a (blue) and Pheo a (purple) while unfilled solid curves represent the absorption bands at the chlorophyll's Q-band and unfilled dotted curves represent emissions of the Chl a (dotted blue line) and Pheo a (dotted purple line).

\section{Conclusions}

We discussed the roles of chlorophyll ( $a$ and $b$ ) and Pheo a molecules in the fluorescence response of aliquots containing natural pigments extracted from Caesalpinia echinata (Brazil-wood), at distinct times of an emulsion of leaves in methanol. Besides the characterization of these solutions, combining Voigt deconvolution with time-resolved fluorescence technique, we were able to observe a selective inner-filter effect, where the features related to the $\mathrm{Chl}$ a molecule are the most affected among the characteristics of the other pigments. This selectivity is not straightforward because the absorption and fluorescence bands of $\mathrm{Chl} b$ and Pheo a are also significantly overlapped with their absorption Q-bands and should have also had been affected. The deconvolution analysis applied to both absorbance and fluorescence spectrum allowed us to propose an interpretation of this selectivity. The overlapping observed for absorption and emission bands indicates that the main mechanism responsible for the inner-filter in these three species is the reabsorption. However, both $\mathrm{Chl} \mathrm{b}$ and Pheo a presents smaller extinction coefficients at Q-band when compared to $\mathrm{Chl}$ a. Thus, we believe that only for $\mathrm{Chl}$ a the absorbance at the overlapped region is large enough to promote an effective inner-filter effect.

Furthermore, by means of the steady-state absorption and fluorescence spectra, we were able to detect the presence of $\beta$-carotene molecule among the great variety of pigments released in the solutions. According to the literature, this carotenoid can effectively interact with $\mathrm{Chl}$ a and favor electron and energy transfer processes, ${ }^{42,43}$ which would affect the chlorophyll's excited state lifetimes. However, under our experimental conditions, we were not able to observe these possible interactions. We believe that the concentrations of $\beta$-carotene adopted in these cited works ${ }^{42,43}$ are higher than the concentrations extracted herein.

\section{Supplementary Information}

Supplementary information is available free of charge at http://jbcs.sbq.org.br as a PDF file.

\section{Acknowledgments}

The authors are indebted with Brazilian agencies: CNPq, FAPESPA, CAPES, PROPESP/UFPA (PAPQ program), and National Institute of Organic Electronic (INEO) for the financial support. 


\section{References}

1. Syafinar, R.; Gomesh, N.; Irwanto, M.; Fareq, M.; Irwan, Y. M.; Energy Procedia 2015, 79, 896.

2. Hug, H.; Bader, M.; Mair, P.; Glatzel, T.; Appl. Energy 2014, 115,216

3. Davies, K. M. In Plant Pigments and Their Manipulation, Annual Plant Reviews; Davies, K. M., ed.; Blackwell Publishing: Oxford, UK, 2004.

4. Hagerthey, S. E.; Louda, J. W.; Mongkronsri, P.; J. Phycol. 2006, 42, 1125.

5. Rodríguez, M.; Mantell, C.; Macías-Sánchez, M.; de la Ossa, E. M.; EJEAFChe, Electron. J. Environ., Agric. Food Chem. 2008, 7, 3150 .

6. Gross, J.; Pigments in Vegetables: Chlorophylls and Carotenoids, $1^{\text {st }}$ ed.; Springer: New York, USA, 1991.

7. Eyster, H. C.; Ohio J. Sci. 1950, 50, 79.

8. Costache, M. A.; Campeanu, G.; Neata, G.; Rom. Biotechnol. Lett. 2012, 17, 7702.

9. Miazek, K.; Ledakowicz, S.; Int. J. Agric. Biol. Eng. 2013, 6, 107.

10. Gouterman, M. In The Porphyrins; Dolphin, D., ed.; Academic Press: New York, USA, 1978.

11. Truscott, T. G.; J. Photochem. Photobiol., B 1990, 6, 359.

12. Rosenberg, J. L.; Annu. Rev. Plant Physiol. 1957, 8, 115.

13. Kouissa, B.; Bouchouit, K.; Abed, S.; Essaidi, Z.; Derkowska, B.; Sahraoui, B.; Opt. Commun. 2013, 293, 75.

14. Corrêa, D. S.; de Boni, L.; dos Santos Jr., D. S.; Barbosa Neto, N. M.; Oliveira Jr., O. N.; Misoguti, L.; Zilio, S. C.; Mendonça, C. R.; Appl. Phys. B: Lasers Opt. 2002, 74, 559.

15. Bouchouit, K.; Derkowska, B.; Migalska-Zalas, A.; Abed, S.; Benali-cherif, N.; Sahraoui, B.; Dyes Pigm. 2010, 86, 161.

16. Zaghdoudi, K.; Ngomo, O.; Vanderesse, R.; Arnoux, P.; Myrzakhmetov, B.; Frochot, C.; Guiavarc'h, Y.; Foods 2017, 6,4 .

17. Kobayashi, M.; Sorimachi, Y.; Fukayama, D.; Komatsu, H.; Kanjoh, T.; Wada, K.; Kawachi, M.; Miyashita, H.; OhnishiKameyama, M.; Ono, H. In Handbook of Photosynthesis, $3^{\text {rd }}$ ed.; Pessarakli, M., ed.; CRC Press, Taylor \& Francis Group. LLC: Florida, USA, 2016.

18. Gouterman, M.; J. Mol. Spectrosc. 1963, 11, 108.

19. Walker Jr., R. D.; Hawkiss, J. E.; J. Am. Chem. Soc. 1952, 74, 4209.

20. Solovchenko, A.; Photoprotection in Plants: Optical ScreeningBased Mechanisms, $1^{\text {st }}$ ed.; Springer: Heidelberg, Germany, 2010.
21. Voicescu, M.; Ionescu, S.; Gatea, F.; J. Fluoresc. 2014, 24, 75.

22. Bastos, I. V. G. A.; da Silva, G. K. C.; Rodrigues, G. C. R.; de Melo, C. M.; Xavier, H. S.; de Souza, I. A.; Rev. Bras. Farm. 2011, 92, 219.

23. Milenković, S. M.; Zvezdanović, J. B.; Anđelković, T. D.; Marković, D. Z.; Adv. Technol. 2012, 1, 16.

24. Di Rocco, H. O.; Cruzado, A.; Acta Phys. Pol., A 2012, 122, 666.

25. Kleinegris, D. M. M.; van Es, M. A.; Janssen, M.; Brandenburg, W. A.; Wijffels, R. H.; J. Appl. Phycol. 2010, 22, 645.

26. Kandori, H.; Sasabe, H.; Mimuro, M.; J. Am. Chem. Soc. 1994, 116, 2671.

27. Ahmed, S. A.; Zang, Z. W.; Yoo, K. M.; Ali, M. A.; Alfano, R. R.; Appl. Opt. 1994, 33, 2747.

28. Terjung, F.; Z. Naturforsch., C: J. Biosci. 1998, 53, 924.

29. Birks, J. B.; J. Phys. B: At. Mol. Phys. 1970, 12, 1704.

30. Sakai, Y.; Kawahigashi, M.; Minami, T.; Inoue, T.; Hirayama, S.; J. Lumin. 1989, 42, 317.

31. Boardman, N. K.; Thorne, S. W.; Biochim. Biophys. Acta, Bioenerg. 1971, 253, 222.

32. Thorne, S. W.; Newcomb, E. H.; Osmond, C. B.; Proc. Natl. Acad. Sci. U. S. A. 1977, 74, 575.

33. White, R. C.; Jones, I. D.; Gibbs, E.; Butler, L. S.; J. Agric. Food Chem. 1972, 20, 773.

34. Pfarrherr, A.; Teuchner, K.; Leupold, D.; Hoffmann, P.; J. Photochem. Photobiol., B 1991, 9, 35.

35. Goedheer, J. C.; Biochim. Biophys. Acta 1964, 88, 304.

36. Lakowicz, J. R.; Principles of Fluorescence Spectroscopy, $3^{\text {rd }}$ ed.; Springer: New York, USA, 2006.

37. Valeur, B.; Berberan-Santos, M. N.; Molecular Fluorescence: Principles and Applications, $2^{\text {nd }}$ ed.; Wiley-VCH: Weinheim, Germany, 2012.

38. Kaplanova, M.; Cermak, K.; J. Photochem. 1981, 15, 313.

39. Kaplanova, M.; Parma, L.; Gen. Physiol. Biophys. 1984, 3, 127.

40. Volkov, A. G.; Markin, V. S.; Leblanc, R. M.; Gugeshashvili, M. I.; Zelent, B.; Munger, G.; J. Solution Chem. 1994, 23, 223.

41. Porra, R. J.; Thompson, W. A.; Kriedemann, P. E.; Biochim. Biophys. Acta, Bioenerg. 1989, 975, 384.

42. Li, T.; Zhang, Y.; Gong, N.; Li, Z.; Sun, C.; Men, Z.; Int. J. Mol. Sci. 2016, 17, 978.

43. Chen, C.; Gong, N.; Li, Z.; Sun, C.; Men, Z.; Molecules 2017, 22,1585

Submitted: February 11, 2019

Published online: July 2, 2019 